Full Length Article

\title{
Double matrix effect in Low Energy Ion Scattering from La surfaces
}

\author{
Andrey A. Zameshin ${ }^{a, *}$, Andrey E. Yakshin ${ }^{a}$, Jacobus M. Sturm ${ }^{\text {a }}$, Hidde H. Brongerma ${ }^{\text {, }}$ \\ Fred Bijkerk ${ }^{\mathrm{a}}$
}

a Industrial Focus Group XUV Optics, MESA+ Institute for Nanotechnology, University of Twente, Enschede, The Netherlands

${ }^{\mathrm{b}}$ Calipso BV, Waalre, The Netherlands

\section{A R T I C L E I N F O}

\section{Article history:}

Received 20 September 2017

Revised 10 January 2018

Accepted 20 January 2018

Available online 2 February 2018

\section{Keywords:}

Low Energy Ion Scattering

LEIS

Lanthanum

Matrix effect

Oscillatory ion yield

Work function

\begin{abstract}
A B S T R A C T
Low Energy Ion Scattering (LEIS) has been performed on several lanthanum-based surfaces. Strong subsurface matrix effects - dependence of surface scattered $\mathrm{He}+$ ion yield on the composition of subsurface layer - have been observed. The ion yield of $\mathrm{He}+$ scattered by La differed by a factor of up to 2.5 for different surfaces, while only the La peak was visible in the spectra. To study these effects and enable surface quantification, $\mathrm{He}+$ ion yields have been measured in a range of incident $\mathrm{He}+$ energies from 1000 to 7500 $\mathrm{eV}$ for LaB6, La2O3, oxidized La and pure La surfaces. The investigation showed that as many as two simultaneous matrix effects are present, each one driven by a separate charge exchange mechanism. The first one is a resonant neutralization from the conduction band of La to an excited state of the He+ ion. It depends on the work function of the surface, which is lowered significantly when La interacts with $\mathrm{O}$ or $\mathrm{B}$. The second mechanism is quasiresonant charge transfer between bound La levels and $\mathrm{He} 1 \mathrm{~s}$, which creates characteristic oscillations in the energy dependence of ion yields. The exact structure of the oscillations depends on small changes in binding energies of interacting La levels. This is the first time quasiresonant charge transfer is proven to be present in La. It is likely that La 5p orbitals participate in this resonance, which can be the first clear observation of a resonance between $\mathrm{p}$ and $\mathrm{s}$ orbitals in LEIS. This type of resonance was previously believed to be absent because of strong damping. We also demonstrated that despite the complex matrix effect precise measurements over a wide energy range allow quantification of the atomic composition of La-based surfaces.
\end{abstract}

(c) 2018 Elsevier B.V. All rights reserved.

\section{Introduction}

La/B based multilayer mirrors are the most promising candidates for the possible next generation EUV lithography at around $6.7 \mathrm{~nm}$ wavelength. They have excellent theoretical reflectance up to $80 \%$ at $6.7 \mathrm{~nm}[1,2]$, but so far their practical performance has been limited to the value of $64 \%$ [3]. The biggest limiting factor is the width of interfaces forming between the constituting materials, since an interlayer of just a few Angstroms has significant impact on the reflectance. This makes it very important to study and understand the La/B interface formation and layer growth on the atomic level. One of the most suitable techniques for this task is Low Energy Ion Scattering (LEIS) of noble gas ions, which has a unique sensitivity to the topmost atomic layer. This makes it very useful for diffusion and growth studies [4-7], also in combination with X-ray Photoelectron Spectroscopy (XPS). Another advantage of LEIS - easy quantification of surface elemental coverage - comes from the usual absence of matrix effects, meaning that generally

\footnotetext{
* Corresponding author.

E-mail address: a.zameshin@utwente.nl (A.A. Zameshin).
}

the low energy ion yield is independent on the surrounding matrix, i.e. chemical environment of the scattering atom.

However, when applying LEIS to La and La/B systems we found a peculiar behavior of the $\mathrm{He}^{+}$ion yield which complicated the quantification of the surface composition of these systems. Even when La was the only material visible in the first atomic layer, its signal increased dramatically during sputter depth profiling, up to a factor of 2.5. In this paper we study this phenomenon in detail. We discuss different ways to explain such a strong change of the signal: contamination and matrix effects. Initial experiments only allow to assume the presence of matrix effects, but based on the results of variable energy measurements we prove the existence of matrix effects in this system. Moreover, we show that this effect is based on as many as two simultaneous matrix effects present in La-based systems. No LEIS studies concerning matrix effects in La can be found in literature.

\section{Experimental}

Samples used in this study were $\mathrm{La}_{2} \mathrm{O}_{3}, \mathrm{LaB}_{6}$, pure La and B-capped La films of $30-200 \mathrm{~nm}$ thickness. The $\mathrm{La}_{2} \mathrm{O}_{3}$ sample 
consisted of compressed powder purchased from Sigma-Aldrich with purity of $99.9 \%$ stated by supplier. For $\mathrm{LaB}_{6}$ we used a cathode from a Physical Electronics XPS setup. Polycrystalline La and amorphous B thin films were deposited at University of Twente by magnetron sputtering in home designed deposition chamber which allowed in-vacuum sample transfers to XPS and LEIS chambers. Deposition chamber had a base pressure of $1 \times 10^{-10} \mathrm{mbar}$. The target-to-sample distance was $8.6 \mathrm{~cm}$. DC magnetron sputtering with $300 \mathrm{~V}$ and $0.13 \mathrm{~A}$ was used for La. RF magnetron sputtering with a total power of $70 \mathrm{~W}$ and DC bias of $97 \mathrm{~V}$ was used for $\mathrm{B}$. The working gas was Ar, with the pressure for the deposition of La and B being $3.1 \times 10^{-3}$ and $4.3 \times 10^{-3} \mathrm{mbar}$, respectively. Deposition rates of $30 \mathrm{~nm} / \mathrm{min}$ for La and $0.5 \mathrm{~nm} / \mathrm{min}$ for B were controlled by quartz mass balances and calibrated by reconstruction of thicknesses of reference samples from Grazing Incidence X-ray Reflectometry measurements at $\mathrm{Cu} \mathrm{K} \alpha$ wavelength.

LEIS measurements were performed either at University of Twente, Enschede, the Netherlands, or at ION-TOF, Münster, Germany. Both instruments were Qtac ${ }^{100}$ with double toroidal electrostatic analyzers (DTA), base pressures down to $1 \times 10^{-10}$ mbar. The instrument at University of Twente was equipped with an electron impact ion source, and the instrument in Münster had an RF plasma high-brightness ion source. The incidence angle of the ions was normal to the sample surface, and the scattering angle was $145^{\circ}$. All variable ion energy measurements were done at the Müster setup due to a better ion gun focusing and broader ion energy range $(1000-7500 \mathrm{eV})$. All in-vacuo studies of asdeposited surfaces were done at University of Twente, with time delay between deposition and measurement being $15 \mathrm{~min}$ for both XPS and LEIS. Whenever sample sputtering was done, $\mathrm{Ar}^{+}$ions with $500 \mathrm{eV}$ were used at an angle of $59^{\circ}$ with respect to the surface normal. Surface charging effect was always excluded by showering poorly conducting surfaces $\left(\mathrm{La}_{2} \mathrm{O}_{3}\right.$ powder) with low energy electrons.

XPS measurements were done with a Thermo Theta Probe spectrometer using $\mathrm{Al} \mathrm{K} \alpha$ radiation. Base pressure in the XPS chamber was $5 \times 10^{-10}$ mbar. Collection and analysis of XPS data was performed in Avantage software [8]. Background was subtracted according to Shirley model, and peak fitting was performed with a product of Gaussian and Lorentzian shapes. Initial quantification was based on Scofield sensitivity factors [9] and a model of uniform elemental distribution.

\section{Results}

Three different LEIS sputter depth profiles of La films are presented in Fig. 1. The first profile was made for a fresh asdeposited $30 \mathrm{~nm}$ La film. The second profile was obtained from the same sample after it was left in $10^{-10}$ mbar vacuum for $24 \mathrm{~h}$, where part of its surface became La oxide and La hydroxide. The third profile was made from a $30 \mathrm{~nm}$ La film capped by $1 \mathrm{~nm}$ B film, which is not enough to form a closed surface. An example of spectra evolution for the third profile is presented in Fig. 2. Other elements like $\mathrm{B}$ and $\mathrm{O}$ were only present in the very beginning of sputtering process, as indicated in Fig. 1 by using open symbols for the La signal. This is also the case for contaminated La film. In the fresh La film no contaminant peaks were seen from the very beginning. In addition to that, the exponential background at lower energies, caused by the sputtering of light elements, is absent in all the LEIS spectra in this study. For example, for the spectra in Fig. 2 the sputtered signal does not even begin to appear at $500 \mathrm{eV}$. This indicates the absence of any noticeable amounts of hydrogen on the surface.

The observed change of the La signal by 2.5 times in absence of other elements is very intriguing and is unusual for LEIS. There are

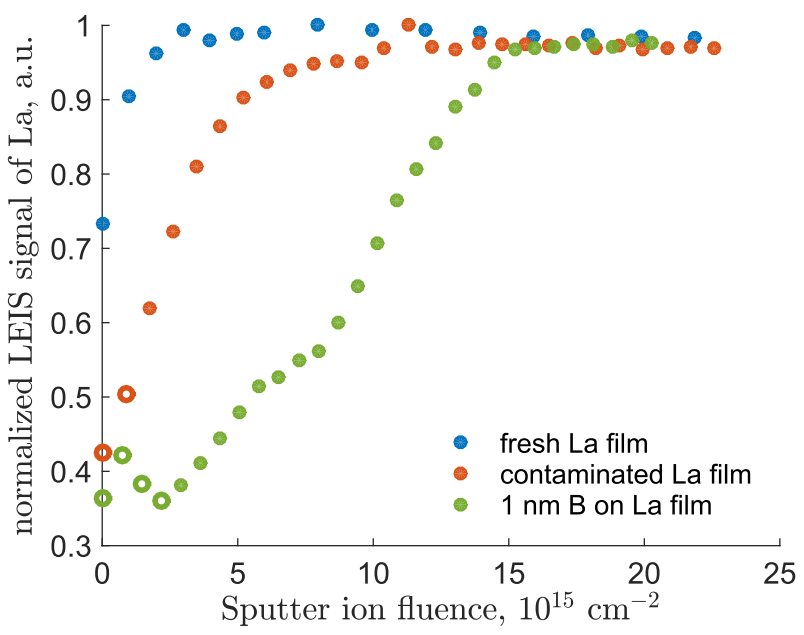

Fig. 1. $3000 \mathrm{eV}{ }^{4} \mathrm{He}^{+}$LEIS depth profiles of La-based thin films: fresh La film, contaminated La film (kept for $24 \mathrm{~h}$ in LEIS main chamber, therefore has $\mathrm{La}, \mathrm{La}_{2} \mathrm{O}_{3}$ and $\mathrm{La}(\mathrm{OH})_{3}$ on the surface), and fresh film of $1 \mathrm{~nm}$ of $\mathrm{B}$ on La. The filled circles are used when only a pure La was present in the spectrum. The open circles are used when some other element is visible (for example see Fig. 2). The sputter rate for B is by an order of magnitude smaller than one for La, therefore to avoid problems with transition compounds all depth profiles are shown for $500 \mathrm{eV} \mathrm{Ar}^{+}$sputter ion fluence.

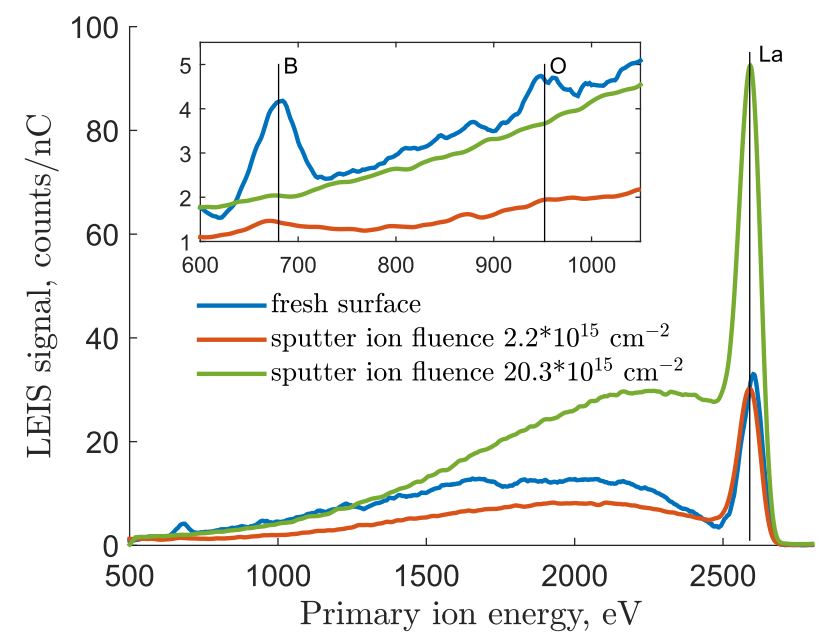

Fig. 2. The evolution of $3000 \mathrm{eV}^{4} \mathrm{He}^{+}$LEIS spectra for a system of $1 \mathrm{~nm} \mathrm{~B}$ on top of $30 \mathrm{~nm}$ La. The spectra are shown for the fresh as-deposited surface and surfaces after sputtering with $500 \mathrm{eV} \mathrm{Ar}{ }^{+}$ion fluences of $2.2 \times 10^{15}$ ions $/ \mathrm{cm}^{2}$ and $20.3 \times 10^{15}$ ions $/ \mathrm{cm}^{2}$. The first sputter ion fluence is the point where peaks of $\mathrm{B}, \mathrm{C}$, and $\mathrm{O}$ become smaller than detection limit. The sputtered ion background at lower energies is absent, which means the absence of any noticeable amount of hydrogen on the surface.

only a few of matrix effects which have a stronger influence on the signal, for example matrix effect in graphitic carbon [10]. Therefore a logical check if this behavior can be explained by small quantities of other elements was performed, shown in Fig. 3 for a binary B/La system. A $3000 \mathrm{eV} \mathrm{He}^{+}$LEIS depth profile was obtained from a thin film of $4 \mathrm{~nm}$ B on top of $200 \mathrm{~nm}$ La. Then B and La ion yields were plotted against each other to check for matrix effects. Several first spectra were excluded due to a presence of a third element - O. During the depth profile, the surface changed from pure $B$, via a $\mathrm{B} / \mathrm{La}$ compound, to pure La. A linear dependence is expected in the absence of matrix effects. Strong deviations from a straight line are observed, which hints towards the presence of a matrix effect. However, this is not a proof, but an indication to start looking into possible matrix effects in this system, involving variable energy 


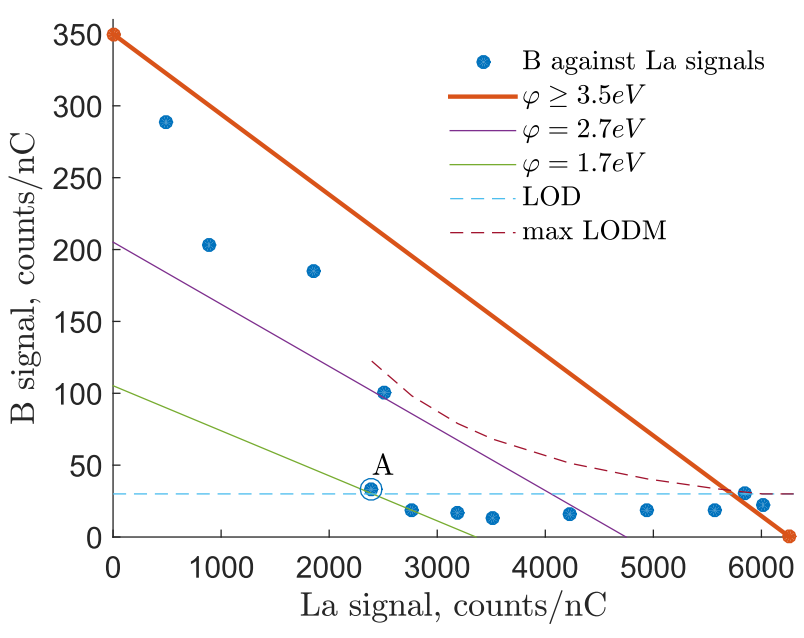

Fig. 3. LEIS signals of $B$ and La plotted against each other for $3000 \mathrm{eV} \mathrm{He} \mathrm{e}^{+}$LEIS depth profiling of the sample with a thin film of $4 \mathrm{~nm}$ B on top of $200 \mathrm{~nm}$ La (blue dots). The red thick line represents a linear dependence, expected in the absence of matrix effects (work function $\varphi \geqslant 3.5 \mathrm{eV}$ ). It connects the LEIS signals of sputtered reference samples of B and La (red dots). The detection limit for B peak of 30 counts/ $\mathrm{nC}$ is shown as a blue dashed line. Three remaining curves and point $\mathrm{A}$ are described in detail in the section "Matrix effect in C-RN". An upper estimation of the limit of detection for $B$ in the presence of a matrix effect (LODM) is shown as a red dashed curve. Linear dependencies, expected for constant work functions of 2.7 and $1.7 \mathrm{eV}$ are shown as thin purple and green lines. (For interpretation of the references to color in this figure legend, the reader is referred to the web version of this article.)

measurements. Further down in this chapter we will present the measurements, while their detailed analysis will be given in Section 4.

Matrix effects can only exist if more than one element is present (carbon with its vastly different valence zone structure of allotropes is again an exception [10]). But LEIS spectra of fresh La film show no sign of contamination. To check for presence of other elements, XPS depth profiles were made for a freshly deposited La film and a contaminated La film, left in XPS main chamber for $24 \mathrm{~h}$. Only La and O peaks were present in the spectra, no other element surpassed the detection limit. The depth profiles for La $4 \mathrm{~d}$ and 0 1s for both samples are shown in Fig. 4(a), quantified for a model of homogeneous elemental distribution. The depth profiles confirm that the bulk of La is pure, but show that the untreated surface of fresh La sample is partially oxidized. O 1 s peaks before sputtering are shown in Fig. 4(b). For a fresh sample the oxygen peak height is at the detection limit, only 2 times higher than the noise level. But the main oxygen state corresponds to La oxide for both fresh and oxidized samples. The transfer time and vacuum conditions in LEIS and XPS chambers are rather similar. Therefore a comparison of LEIS and XPS spectra is possible, which allows for the following conclusion. Since for the fresh La sample LEIS shows only La, and XPS shows the presence of $\mathrm{O}$, it means that $\mathrm{O}$ is present in the second atomic layer, where it is undetectable with LEIS. Similar effect was observed for different transition metals - Ti, Nb, $\mathrm{Zr}$, Mo [11]. Therefore the possibly observed matrix effect in LEIS can be called a subsurface matrix effect. The same holds for B and La interaction - during sputtering process B is quickly moved to the second atomic layer, both due to strong preferential sputtering and surface segregation of La. Such an atomic arrangement in $\mathrm{La}_{2} \mathrm{O}_{3}$ and $\mathrm{LaB}_{6}$ molecules will lead to the formation of a dipole layer at the surface, which in turn will change the work function of the surface. Work function dependent matrix effects are known in LEIS [12], for example in Ba/W system. The present situation can be similar.

Atomic concentrations in Fig. 4(a) are only valid for uniform distribution model. For a fresh surface before sputtering this model gives $10.4 \%$ oxygen. Within a model of subsurface oxygen, we can estimate a possible atomic concentration of oxygen in the second atomic layer only. For this purpose we can represent the sample as a trilayer model: bottom La layer of infinite thickness, intermediate layer of thickness $d_{2}$, with $\mathrm{O}$ concentration $c_{2}$ and La concentration $1-c_{2}$, and top layer of thickness $d_{1}$, consisting of La atoms only. If intensity expected from infinitely thick sample with $O$ concentration $c_{\infty}$ is $c_{\infty} I_{\infty}$, we can represent intensity from second layer through a combination of two attenuation factors [13]: $c_{2} I_{\infty}\left(1-\exp \left(-\frac{d_{2}}{\lambda \cos \theta}\right)\right) \exp \left(-\frac{d_{1}}{\lambda \cos \theta}\right)$, where $\lambda$ is an attenuation length and $\theta$ is an escape angle. Therefore the ratio between atomic concentration of oxygen in the uniform distribution model $c_{\infty}$ and in the trilayer model $c_{2}$ is

$\frac{c_{\infty}}{c_{2}}=\left(1-\exp \left(-\frac{d_{2}}{\lambda \cos \theta}\right)\right) \exp \left(-\frac{d_{1}}{\lambda \cos \theta}\right)$.

For a simple estimation we can assume $d_{1}$ and $d_{2}$ to be equal to a double of covalent radii of La and $\mathrm{O}$ atoms, which are 0.207 and $0.066 \mathrm{~nm}$ correspondingly. Inelastic mean free path $\lambda$ of electrons

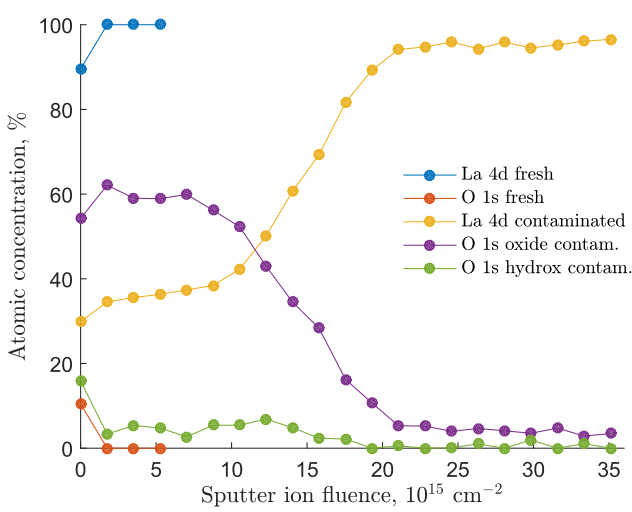

(a)

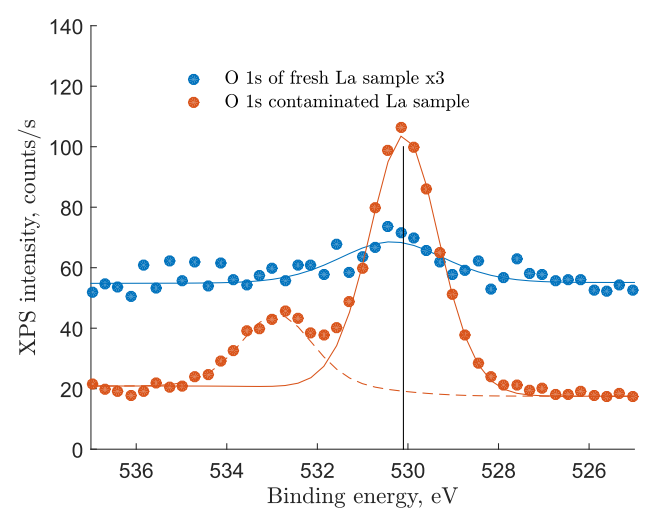

(b)

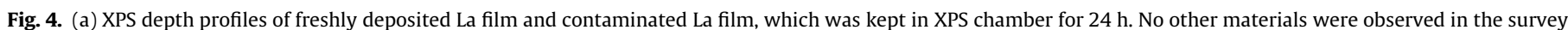

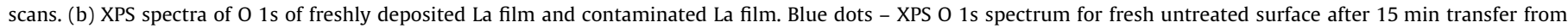

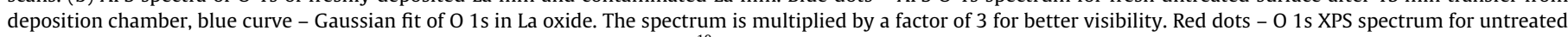

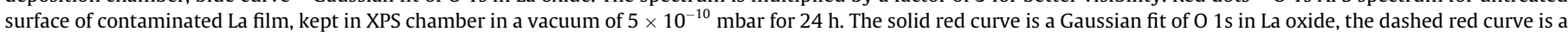

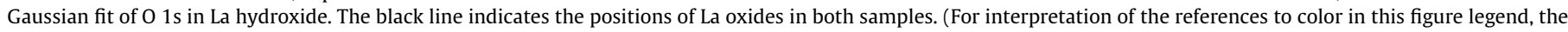
reader is referred to the web version of this article.) 
generated from 0 1s levels and escaping through La layer was taken as $1.63 \mathrm{~nm}$. XPS measurements were not angular resolved, therefore the data was collected for multiple angles $\theta$ simultaneously. To estimate $c_{2}$, we calculate it for 3 different angles: 30, 45 and $60^{\circ}$. The results are $c_{2}=1.6,1.4$ and 1.2. Keeping in mind that this is only a rough approximation, we consider the model of subsurface oxygen to be plausible.

The matrix effects in LEIS can be studied by varying the primary ion energy, the proper reasoning for which will be presented in Section 4. Several samples were studied with variable primary gun energies at ION-TOF in Muenster. All the surfaces used in this study had to have stable surface composition during approximately $2 \mathrm{~h}$ while multiple energy points were collected. Four surfaces were selected: $\mathrm{La}_{2} \mathrm{O}_{3}$ and $\mathrm{LaB}_{6}$ as references, and pure $\mathrm{La}$ and in-vacuum contaminated La to represent two different surface states of La film observed in Fig. $1 . \mathrm{La}_{2} \mathrm{O}_{3}$ and $\mathrm{LaB}_{6}$ were sputter cleaned after introduction into the vacuum chamber, then left for $30 \mathrm{~min}$. This was a necessary step to stabilize their surface composition through oxidation. Within this time slot oxidation most probably occurs only at the surface active sites. Subsequent measurements did not have a destabilizing effect on the surface composition due to the low $\mathrm{He}^{+}$sputter rates of $\mathrm{B}, \mathrm{O}$ and La. Elemental La was prepared differently. Because of fast destructive oxidation the entire $200 \mathrm{~nm}$ La film would become La oxide powder during the atmospheric transfer to the measurement location. Therefore $200 \mathrm{~nm}$ La film was capped by $4 \mathrm{~nm}$ of B. Then in the LEIS chamber it was sputter cleaned until saturation of the La signal. To measure contaminated La, the exposed surface of La film was left in the vacuum chamber for 1 night. Then it was sputtered by $3 \mathrm{keV} \mathrm{He}^{+}$ions to remove hydrogen contamination from the surface and ensure that the surface is stable. Otherwise $\mathrm{H}$ would be constantly sputtered during the measurement itself, increasing the La atomic surface fraction.

To measure a pure La surface we had to avoid any oxidation. Therefore $\mathrm{Ar}^{+}$sputtering was performed constantly before and during all the measurements, resulting in a "dynamically stable" surface under constant $\mathrm{Ar}^{+}$ion bombardment. Due to the low work function of the La surface, both scattered and sputtered ions are effectively neutralized when leaving the surface. Therefore background signal of the sputtered ions did not influence the signal of the scattered ions in the energy range of interest (above $800 \mathrm{eV}$ ).

For every measurement the La peak was separated from the indepth tail and integrated. The energy dependencies of La peaks for the four different abovementioned surfaces are shown in Fig. 5. The error bars include ion current instabilities and peak fitting errors.

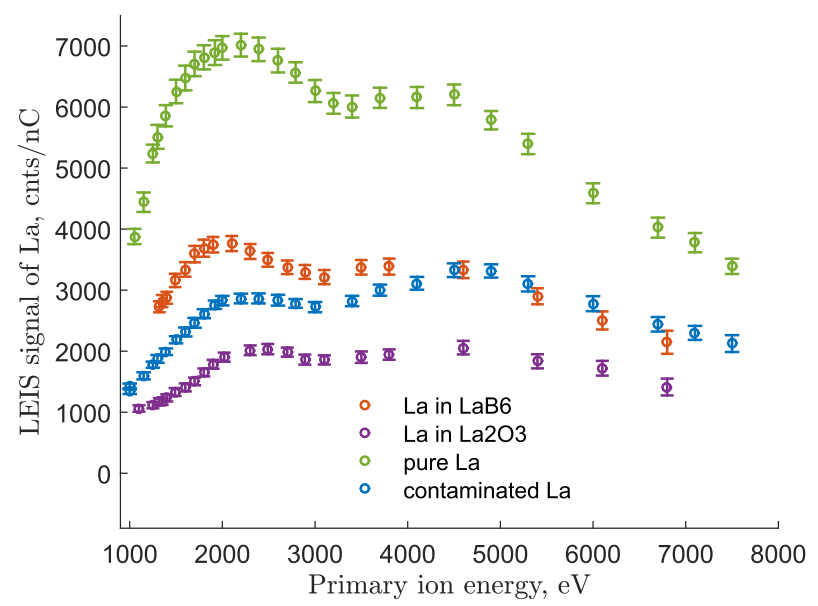

Fig. 5. Energy dependence of ${ }^{4} \mathrm{He}^{+}$LEIS yield of La in different chemical environment - in $\mathrm{LaB}_{6}, \mathrm{La}_{2} \mathrm{O}_{3}$, in-vacuum contaminated La samples, and pure La.

\section{Discussion}

\subsection{Ion yield in LEIS}

The total amount of detected ions $Y_{i}$ scattered from an element $i$ can be described as [14]:

$Y_{i}=\frac{I_{p} t}{e} \xi R P_{i}^{+} \frac{d \sigma_{i}}{d \Omega} N_{i}$

where $I_{p}$ is the primary ion current, $t$ is the measurement time, $e$ is the electron charge, $\xi$ is the analyzer + detector instrumental factor, $R$ is a roughness factor, $P_{i}^{+}$is the ion fraction after scattering by element $i, \frac{d \sigma_{i}}{d \Omega}$ is the differential cross-section in $\AA^{2} / \mathrm{sr}, N_{i}$ is the atomic surface concentration in $\mathrm{cm}^{-2}$. $Y_{i}$ is called an ion yield in LEIS literature. In LEIS experiments, however, the signal or ion yield is often expressed as

$S_{i}=\frac{Y_{i}}{I_{p} t}=\frac{1}{e} \xi R P_{i}^{+} \frac{d \sigma_{i}}{d \Omega} N_{i}$

which is measured in counts/nC. We do not want to change the conventional definitions in this paper, and to distinguish these two different terms will call $Y_{i}$ an ion yield and $S_{i}$ a LEIS signal. However, in most of the cases these terms are used interchangeably, and the difference is only important when exact dimensions of an "ion yield" play a role.

The large increase of La signal observed in Fig. 1 has to be explained by Eq. (3). There are several variables in this formula that can potentially change during the depth profiling: $I_{p}, R, N_{i}, P_{i}^{+}$.

If the ion current $I_{p}$ is measured only once before making a depth profile, long-term drifts or short-term fluctuations of $I_{p}$ can influence the behavior of $S_{L a}$ in the depth profile. Therefore separate experiments with additional re-measurement of $I_{p}$ were done, and potential influence of this factor was limited only to a small error.

The roughness factor $R=1$ for ideally flat surfaces and $R<1$ for rough surfaces due to geometrical effects such as shadowing and blocking, as well as grazing incidence neutralization [15]. However even for a very well-developed surfaces like for catalysts $[15,16] R$ value is bigger than 0.6 , and the effect in Fig. 1 would only be explained by a change of $R$ from 0.4 to 1 . This is impossible to achieve in any conventional thin film, and would require some initial extreme columnar growth followed by complete smoothening during sputtering. This is not the case for La samples in this study. RMS roughness measured by atomic force microscopy on atmosphere-exposed $100 \mathrm{~nm}$ La films with $2 \mathrm{~nm} \mathrm{Ru}$ cap is 0.27 $\mathrm{nm}$, which excludes extreme growth modes.

The next factor to consider is $N_{i}$. Small amounts of other elements, i.e. $\mathrm{B}, \mathrm{O}$ and $\mathrm{C}$ can be present at the surface below the limit of detection (LOD), therefore decreasing the visible surface coverage of La at the beginning of sputtering. If the major reason for the initial drop of the La signal is contamination and not a matrix effect, then reference values of $\mathrm{B}$ and $\mathrm{O}$ can be used to determine their surface coverages $c_{i}=\frac{N_{i}}{N_{i}^{r e f}}$ at the LOD $c_{B}^{L O D}$ and $c_{O}^{L O D}$. Assuming that the minimal detectable peak area is 30 counts/nC (example of Fig. 2), we used reference samples of pure boron and boron oxide to obtain $c_{O}^{L O D}=6.4 \%$ and $c_{B}^{L O D}=8.4 \%$. Therefore this effect could only possibly explain a change in La surface coverage of $15 \%$ in case of a B-capped La film, and only $6.5 \%$ in case of a fresh La film. The observed changes are larger, which means that we should also look for other mechanisms responsible for changes of the LEIS signal, while keeping in mind that the effect of contamination cannot be excluded as well. The presence of noticeable amounts of $C$ was ruled out by XPS measurements, where it was neither detected 
for fresh La surface, nor for the in vacuum contaminated La surface. We estimate detection limit of surface coverage of $C$ by XPS as $10 \%$.

The last factor is $P_{L a}^{+}$. Survival probability of a scattered $\mathrm{He}^{+}$ion strongly depends on the details of charge-exchange processes neutralization and reionization - happening between this ion and the surface of a sample. In the LEIS field four general mechanisms of charge transfer are recognized [14]: Auger neutralization of several types (AN), resonant neutralization from the conduction band of a target to an excited state of a projectile (C-RN), (quasi-) resonant charge transfer between bound levels of a target and the ground state of a projectile (qRCT) and collision-induced neutralization and reionization (CIN/CIR), also called resonant neutralization/reionization at close collision. These mechanisms result in a different energy dependencies of the ion survival probability $P^{+}$.

AN and C-RN are one-directional mechanisms (only neutralization), therefore one can write a simple rate equation for them, which leads to an exponential dependence of $P^{+}$on ion velocity $[12,14]$, described by the Hagstrum [17] and Godfrey-Woodruff [18] models:

$P^{+}=\exp \left(-v_{c} \frac{1}{v}\right)$

here $v_{c}$ is an integrated electron transfer rate in $\mathrm{m} / \mathrm{s}$, called characteristic velocity [14], $\frac{1}{v}$ can represent inversed incident ion velocity $\frac{1}{v_{0}}+\frac{1}{v_{f}}$ for the Godfrey-Woodruff model or $\frac{1}{v_{0, \perp}}+\frac{1}{v_{f, \perp}}$ for the Hagstrum model, $v_{0}$ and $v_{f}$ are incoming and scattered ion velocities correspondingly, and $v_{0, \perp}$ and $v_{f, \perp}$ are surface normal components of these velocities. Since both AN and C-RN involve conduction band, they are not local and therefore follow the Hagstrum model. AN is always present, but since it is a two-electron process, its transition rates are usually lower than that of C-RN, if the second one takes place. C-RN depends on a work function of a surface $\varphi$ and becomes significant (or even dominant) if $\varphi$ is lower than $\varphi \approx 3.5 \mathrm{eV}$ [19]. Therefore for low $\varphi$ materials C-RN leads to a work function related matrix effect.

CIN/CIR occurs when the incident ion energy exceeds a threshold energy. Collision-induced neutralization is stronger than reionization therefore its appearance can be indicated by a visible "break" in the slope of energy dependence of the ion yield $[20,21]$. Sometimes no break is present [22]. CIN follows the Godfrey-Woodruff model above the reionization threshold. Our measurement range is above the La reionization threshold [23], and the presence of CIN/CIR should contribute to the absolute value of $v_{c}$, but not change it for different La samples. Since no matrix effect in CIN/CIR is expected, we do not discuss it further.

qRCT is the only mechanism to have an oscillatory ion velocity dependence. For small energy mismatch between interacting energy levels the transition rates for neutralization and reionization in qRCT are similar. The charge of the scattered He oscillates between $\mathrm{He}^{0}$ and $\mathrm{He}^{+}$, as a function of interaction time with the target atom, and therefore ion energy. An example of such interaction is the $\mathrm{He}^{+}-\mathrm{Ga}$ resonance [24]. When He 1s is (quasi-)resonant with two or more electronic states, a band of energy states, or has a large mismatch with the interacting level, quasiresonant neutralization ( $q R N$ ) becomes much stronger than quasiresonant reionization, which leads to damping of the oscillations $[25,26,10]$. In the case when qRCT becomes mostly qRN, as a one-directional process it will also have a characteristic velocity of its own.

The existing knowledge in the field of LEIS is that the presence of qRCT requires two conditions [14]. The first is a close energy match between levels of a projectile ion and bound levels of the target atoms. Normally, energy mismatches less than $5 \mathrm{eV}$ lead to strong resonances, while weak resonances are still observed until $10 \mathrm{eV}[24]$. The second condition is the same symmetry of interact- ing orbitals - strong resonances with $\mathrm{He}^{+}$ions have so far only been observed for $\mathrm{s} \leftrightarrow \mathrm{d}$ transitions [24].

From these four mechanisms C-RN and qRCT can depend on the chemical environment. For the low work function surfaces $v_{c}$ of CRN depends on the work function, while the structure of oscillations in qRCT also depends on the binding energy of interacting orbitals, which can shift in different compounds. Further we will discuss application of both of these mechanisms to our La-based systems.

\subsection{Matrix effects in C-RN}

$\mathrm{LaB}_{6}$ is a known cathode material, one of the reasons being its intrinsic low work function, which is $2.3-2.5 \mathrm{eV}$ for (100) surface, 2.5 for (110) and $3.3 \mathrm{eV}$ for (111) surface [27,28]. For (100) and (110) surfaces the positively charged La ions are positioned above negatively charged $\mathrm{B}$ ions, which creates a dipole moment and lowers the work function. $\mathrm{La}_{2} \mathrm{O}_{3}$ is also used as small additions to tungsten cathodes $[29,30]$, where in small quantities it helps to achieve surfaces with $\varphi$ as low as $2 \mathrm{eV}$ [31]. The calculated values for pure $\mathrm{La}_{2} \mathrm{O}_{3}$ are as low as $1.59 \mathrm{eV}$ [32]. There may be two reasons for lowering the work function in the lanthanum oxide: dipole formation with negative $\mathrm{O}$ ions in the second layer, which correlates with our proposed subsurface matrix effect, or low density of free electrons in the surface layer [32]. Pure La has a work function around $3.5 \mathrm{eV}$ [33]. Since we do not deal with single crystals and also apply sputtering of our samples, the surfaces used in this study will not have the same $\varphi$ values as listed above. Unfortunately we were not able to measure work functions of our exact surfaces, therefore we had to assume literature values. These values are used for qualitative purposes only.

There is an established procedure of analysis of matrix effect in C-RN $[34,19,12]$. Assuming the Hagstrum model for C-RN, we can combine Eqs. (4) and (3) to make a coordinate set in which the energy dependence of the LEIS signal will look linear:

$\ln \left(\frac{S_{i}}{\frac{d \sigma_{i}}{d \Omega}}\right)=-v_{c}\left(\frac{1}{v_{0, \perp}}+\frac{1}{v_{f, \perp}}\right)+\ln \left(C \times N_{i}\right)$,

$C=\frac{\xi R}{e}$.

Here $v_{c}$ serves as a slope and $\ln \left(C \times N_{i}\right)$ as vertical offset of the line. Ideally this allows to determine both $v_{c}$ and $N_{i}$, as it was done elsewhere for Ba $[34,19,12]$. Differential cross-sections of $\mathrm{He}^{+}$scattering by La are calculated using the universal ZBL potential [35]. Then the data from Fig. 5 are replotted as $\ln \left(\frac{s_{i}}{\frac{d \sigma_{i}}{d \Omega}}\right)$ versus $\frac{1}{v_{0, \perp}}+\frac{1}{v_{f, \perp}}$ in Fig. 6. We see that least square linear fitting results in a quite good fit in the whole inversed velocity range. Additional structuring of the data does not change the overall linear trend and will be discussed in details in the next section.

The lines for different surfaces have different slopes and vertical offsets. From Eq. (5) the slope values represent $v_{c}$, and the vertical offsets are $\ln \left(C \times N_{L a}\right)$. Extracted $v_{c}$ and $N_{L a}$ values are presented in Table 1 together with their standard deviations. Since $C$ is an unknown constant, determination of $N_{L a}$ is only relative to the surface of the purest La reference sample. We can estimate a value of $N_{L a}$ for a close packed surface plane as $N_{L a}=\left(\frac{\rho_{L a} N_{A v}}{M_{L a}}\right)^{2 / 3}=0.90 \times 10^{15} \mathrm{~cm}^{-2}$, where $\rho_{L a}$ is bulk La density, $N_{A v}$ - Avogadro's number, $M_{L a}$ - molar mass of La. Then atomic surface concentrations of $\mathrm{La}$ in $\mathrm{LaB}_{6}$ and $\mathrm{La}_{2} \mathrm{O}_{3}$ compounds are about $60 \%$ of that value. Due to the fact that the sample preparation involved $\mathrm{Ar}^{+}$sputtering, the top surfaces are amorphous and we cannot assign any crystal orientation to them. The in-vacuum con- 


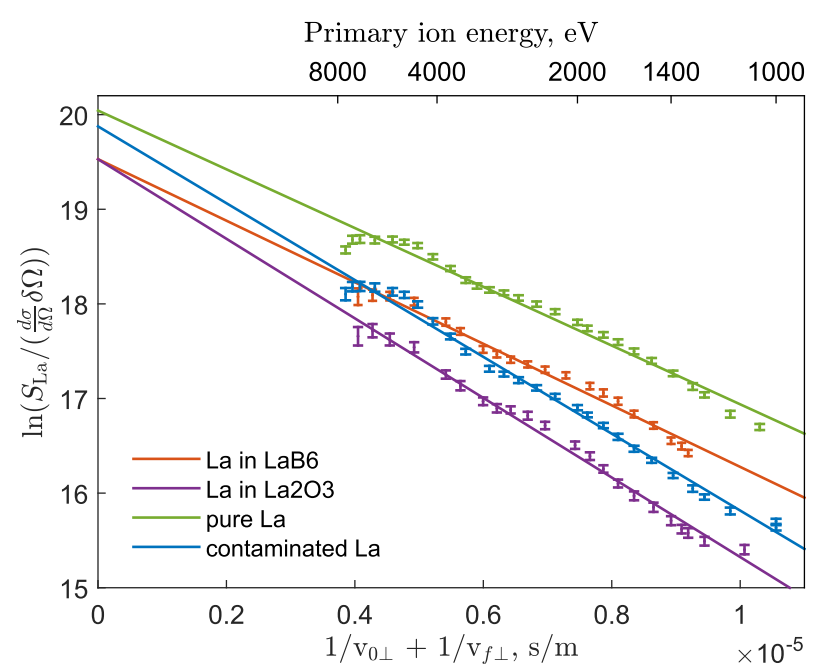

Fig. 6. Dependence of logarithmic normalized LEIS signal of La in different samples on the inversed velocity of $\mathrm{He}^{+}$ions, following Eq. (5). The least square fitted lines are shown for each set. The linear slope is $v_{c}$, the vertical offset is $\ln \left(C \times N_{L a}\right)$. $S_{L a}$ is in counts/nC, $\frac{d \sigma_{i}}{d \Omega} \delta \Omega$ (differential cross-section multiplied by analyzer acceptance angle) is in $\AA^{2}$.

Table 1

Characteristic velocities and relative atomic surface concentrations of La in different compounds. All samples have amorphous top surface layer due to $\mathrm{Ar}^{+}$sputtering.

\begin{tabular}{cccc}
\hline Sample & $\begin{array}{c}v_{c} \text { of } \mathrm{He}^{+} \text {on } \\
\mathrm{La}, 10^{5} \mathrm{~m} / \mathrm{s}\end{array}$ & $\begin{array}{c}N_{L a} \text { relative } \\
\text { (surface coverage) }\end{array}$ & $\begin{array}{c}N_{L a} \text { estimated, } \\
10^{15} \mathrm{~cm}^{-2}\end{array}$ \\
\hline pure $\mathrm{La}$ & $3.1 \pm 0.10$ & 1 & 0.90 (assum.) \\
contam. $\mathrm{La}(\mathrm{La}+$ & $4.06 \pm 0.08$ & $0.85 \pm 0.08$ & $0.76 \pm 0.07$ \\
$\left.\mathrm{La}_{2} \mathrm{O}_{3}+\mathrm{La}(\mathrm{OH})_{3}\right)$ & & & \\
$\mathrm{LaB}_{6}$ & $3.3 \pm 0.10$ & $0.60 \pm 0.06$ & $0.54 \pm 0.05$ \\
$\mathrm{La}_{2} \mathrm{O}_{3}$ & $4.2 \pm 0.10$ & $0.60 \pm 0.06$ & $0.54 \pm 0.05$ \\
\hline
\end{tabular}

taminated La sample has a surface coverage of La of $85 \%$, so the contamination is only responsible for $15 \%$ of the decrease of the La ion yield.

Although $\mathrm{La}$ in $\mathrm{La}_{2} \mathrm{O}_{3}$ and $\mathrm{LaB}_{6}$ has almost the same surface concentration, $v_{c}$ values are very different. The same applies to comparison of pure and contaminated La surfaces. This significant difference between characteristic velocities is the most definite proof of the presence of matrix effects in La-based systems. The difference in characteristic velocities automatically leads to strongly different values of the ion yield for any primary energies in the involved energy range. Characteristic velocity is the lowest for pure La, followed by boride, with contaminated (i.e. oxidized) La and La oxide having the highest $v_{c}$. Applied to the neutralization strength of C-RN it means that the work function scales in the following way: pure La $>$ La boride $>$ La oxide, which agrees well with the abovementioned literature values of $\varphi$. In the work of Cortenraad [12] relative changes in characteristic velocity were independent of ion-target combination: in all cases the characteristic velocity was increasing by roughly $1 \times 10^{5} \mathrm{~m} / \mathrm{s}$ per $1 \mathrm{eV}$ of decrease of work function. Under this assumption we can use Cortenraad's data to calculate relative work functions for our systems. If the work function of pure La $\varphi_{\mathrm{La}}=3.5 \mathrm{eV}$, we obtain $\varphi_{\mathrm{LaB}_{6}} \approx 3.3 \mathrm{eV}$ and $\varphi_{\mathrm{La}_{2} \mathrm{O}_{3}} \approx 2.4 \mathrm{eV}$. These values qualitatively agree with available literature data, but do not match exactly.

Now we can claim that for these four samples the dependence of the La ion yield on its chemical environment - matrix effect can well be ascribed to work function dependent C-RN. It is important to mention that C-RN is not the only neutralization mecha- nism with a characteristic velocity, so part of the obtained values for $v_{c}$ is due to the contributions of AN, CIN/CIR and qRN. They, however, will not exhibit a dependence of $v_{c}$ on work function, so the relative changes between $v_{c}$ are only happening because of C-RN.

The obtained value of $v_{c}$ for pure La can be compared to literature values of Mikhailov [36] for different metals. Taking into account the relative value of $v_{c}$ for $\mathrm{Pd}\left(1.3 \times 10^{5} \mathrm{~m} / \mathrm{s}\right)$, we determine that a large group of metals, such as $\mathrm{Al}, \mathrm{Ta}, \mathrm{W}, \mathrm{Cr}$ and $\mathrm{Mo}$, has $v_{c}$ around $(3.4-3.8) \times 10^{5} \mathrm{~m} / \mathrm{s}$. This can be directly compared to our result if corrected for perpendicular components of inversed velocities, yielding $v_{c}$ around $(3.1-3.4) \times 10^{5} \mathrm{~m} / \mathrm{s}$. So the obtained value of $v_{c}$ for La matches well to other elements with multiple empty valence states. From the onset of the La tail we estimate the reionization threshold for La as $550 \mathrm{eV}$, which also correlates well with $\mathrm{Al}, \mathrm{Ta}, \mathrm{W}, \mathrm{Cr}$ and Mo. It should be noted that the La spectrum in Fig. 2 must not be used for determination of the reionization threshold, because onset of the tail is shifted to higher energies due to the limited La thickness remaining after sputtering.

After this conclusion we can mention the uncertainty of determination of the extracted surface densities/coverages. This uncertainty is actually higher than the value extracted from least square fitting. We know that for low work functions C-RN not only increases the slope, but the vertical offset as well. In the work of Cortenraad $[19,12]$ the vertical offsets of $\mathrm{Ne}^{+}$scattering from Ba were increased dramatically, making quantification almost impossible. In the case of $\mathrm{He}^{+}$ions the overestimation of vertical offset was within $10 \%$. This is due to the lower relative contribution of C-RN to the overall neutralization, which should be strongly influenced by CIN after the reionization threshold. Therefore our surface coverages for surfaces with higher characteristic velocities are overestimated within $10 \%$. This does not affect any conclusions about $\mathrm{LaB}_{6}$, but makes conclusions about $\mathrm{La}_{2} \mathrm{O}_{3}$ less trustworty.

Since C-RN has a non-local nature, the characteristic velocity is the same for the whole surface and not element-specific. Matrix effect caused by C-RN should not only change LEIS signals of La, but also signals of $B$ and $O$. Therefore we also can perform an evaluation of how much $S_{B}$ and $S_{O}$ can change for $3000 \mathrm{eV} \mathrm{He}^{+}$and also revise the estimation of the maximum possible surface coverages of B and O made in the section "Ion yield in LEIS". We introduce $S_{i}^{H}$ and $S_{i}^{L}$ - LEIS signals of element $i$ for a high work function surface and a low work function surface accordingly. If we define $\alpha_{i}$ as their ratio, then using Eq. (3) and (4) we obtain

$$
\begin{aligned}
\frac{S_{i}^{H}}{S_{i}^{L}} \stackrel{\text { def }}{=} \alpha_{i} & =\frac{P_{i}^{+H} N_{i}^{H}}{P_{i}^{+L} N_{i}^{L}}=\frac{N_{i}^{H}}{N_{i}^{L}} \frac{\exp \left(-v_{c}^{H} \frac{1}{v_{i}}\right)}{\exp \left(-v_{c}^{L} \frac{1}{v_{i}}\right)}= \\
& =\frac{N_{i}^{H}}{N_{i}^{L}} \exp \left(\left(v_{c}^{L}-v_{c}^{H}\right) \frac{1}{v_{i}}\right)=\frac{N_{i}^{H}}{N_{i}^{L}} \exp \left(\Delta v_{c}^{L-H} \frac{1}{v_{i}}\right) .
\end{aligned}
$$

Here we use the same denotation of superscript $\mathrm{H}$ and $\mathrm{L}$ to define $P_{i}^{+}, N_{i}$ and $v_{c}$ for high and low work function. In the Hagstrum model $\frac{1}{v_{i}}=\frac{1}{v_{0, \perp}}+\frac{1}{v_{f, \perp}}$. $\Delta v_{c}^{L-H}$ is the difference between characteristic velocities for surfaces with low and high work function. Since we only care about a region where $N_{L a}^{H} \approx N_{L a}^{L}$, with a help of Eq. (6) we estimate $\frac{S_{B}^{H}}{S_{B}^{L}}$ and $\frac{S_{O}^{H}}{S_{O}^{L}}$ :

$$
\begin{aligned}
& \frac{\ln \alpha_{L a}}{1 / v_{L a}}=\frac{\ln \alpha_{B}-\ln \frac{N_{B}^{H}}{N_{B}^{L}}}{1 / v_{B}}=\frac{\ln \alpha_{O}-\ln \frac{N_{O}^{H}}{N_{O}^{L}}}{1 / v_{O}}, \\
& \alpha_{B}=\frac{N_{B}^{H}}{N_{B}^{L}} \exp \left(\frac{v_{L a}}{v_{B}} \ln \alpha_{L a}\right),
\end{aligned}
$$


$\alpha_{O}=\frac{N_{O}^{H}}{N_{O}^{L}} \exp \left(\frac{v_{L a}}{v_{0}} \ln \alpha_{L a}\right)$.

Unfortunately, exact determination of $\alpha_{B}$ and $\alpha_{0}$ requires knowledge of the surface coverages of these elements. In approximation of constant surface composition and only variable work function $\alpha_{B}=\exp \left(\frac{v_{L a}}{v_{B}} \ln \alpha_{L a}\right)$ and $\alpha_{O}=\exp \left(\frac{v_{L a}}{v_{0}} \ln \alpha_{L a}\right)$. In the real situation we know that presence of $B$ and $O$ is the cause of lower work function, therefore $N_{B}^{H} \leqslant N_{B}^{L}$ and $N_{O}^{H} \leqslant N_{O}^{L}$. This sets

$\alpha_{B} \leqslant \exp \left(\frac{v_{L a}}{v_{B}} \ln \alpha_{L a}\right)$

$\alpha_{O} \leqslant \exp \left(\frac{v_{L a}}{v_{0}} \ln \alpha_{L a}\right)$

Now for $3000 \mathrm{eV} \mathrm{He}{ }^{+}$using $\alpha_{B}$ and $\alpha_{O}$ we can estimate how $S_{B}$ and $S_{O}$ change compared to $S_{L} a$. While we don't see the peaks of $\mathrm{B}$ and $\mathrm{O}$, we can still account for this change by introducing an effective limit of detection in presence of matrix effects (LODM), which is the normal LOD multiplied by $\alpha_{i}$. We calculated the upper estimation of LODM for B in Fig. 3, where it is shown together with LOD. All the points which were below LOD are still below LODM, except for the last one. The upper estimation for LODM is no longer applicable when we already see that B signal is strongly increasing.

In the section "Ion yield in LEIS" we obtained surface coverages of $\mathrm{B}$ and $\mathrm{O}$ at their detection limits $c_{B}^{L O D}=8.4 \%$ and $c_{O}^{L O D}=6.4 \%$. Now we can estimate the maximal LODM for them in presence of the matrix effect caused by C-RN. The scaling factors $\alpha_{L a}$ are extracted from Fig. 1 as the maximal drop of $S_{L a}$ in the absence of detectable amounts of other elements: 2.5 for the B-capped La film, 1.57 for contaminated La film and 1.36 for fresh La film. Then we obtain $c_{B}^{L O D M} \leqslant 34.0 \%$ and $c_{O}^{L O D M} \leqslant 20.9 \%$ for the B-capped La film, $c_{O}^{L O D M} \leqslant 11.5 \%$ for the contaminated La film, and $c_{O}^{L O D M} \leqslant 9.5 \%$ for the fresh La film. For all three samples this unseen contamination can potentially lower $N_{L a}$ and therefore $S_{L a}$ too, but it must be noted that this cannot be the only factor for the decrease of $S_{L a}$, because only the presence of the matrix effect allows LODM values to be higher than LOD values. Without the matrix effect LODs alone are not enough to explain the observed effect.

Eq. (6) can also help to understand Fig. 3 better. The expected linear dependence in absence of matrix effects is based on two points: the reference signals of La and B. Applying Eq. (6) to these values, we can plot an expected linear dependence for any fixed value of $\Delta v_{c}^{L-H}$, which is connected to $\varphi$ through our previous estimation of $1 \times 10^{5} \mathrm{~m} / \mathrm{s}$ per $1 \mathrm{eV}$ change of work function. Using the constant $\varphi$ lines we can estimate the range of work functions that is needed to explain the experimental data in Fig. 3. Almost all of the points can be explained by $\varphi>2.7 \mathrm{eV}$. The points with no detected $B$ signal are explained by the same work function, because their maximal possible $B$ concentration is given by the LODM curve. As mentioned before, such work functions of La-B compounds can be expected from the literature. However, there is a single point (marked "A" with an additional circle around it) where both $B$ and La signals are very low. If these values are fully due to a low work function, it would require $\varphi$ as low as $1.7 \mathrm{eV}$. Such value seems unlikely. We can speculate that B recoil implantation could lead to an extra low work function, but part of the signal reduction can possibly result from other factors. For example, we can expect a deviation from the Vegard's law. Since the density of $\mathrm{LaB}_{6}$ is different from a linear combination of densities of elemental La and B, the expected linear dependencies for fixed $\varphi$ should be non-linear instead. Small amounts of contamination with other elements and changes in roughness factor $R$ can also reduce La and B signals.

\subsection{Matrix effects in $q R C T$}

The linear fit in Fig. 6 cannot describe all the features of the inversed velocity dependence plots, since there the curves are also visibly structured. This is a very interesting observation to discuss in detail. The original classification paper of Rusch and Erickson [24] reported "structured" ion yields in $200-2000 \mathrm{eV} \mathrm{He}^{+}$ion scattering from a large group of lanthanides ( $\mathrm{La}, \mathrm{Ce}, \mathrm{Nd}, \mathrm{Sm}, \mathrm{Gd}, \mathrm{Dy}, \mathrm{Er}$, $\mathrm{Yb}$ ). Unfortunately no graphs were presented, so the exact shape of the "structuring" effects is unknown. Two currently understood types of structuring are connected to CIN/CIR (break in the slope) and qRCT (oscillation). The effect observed in Fig. 6 looks like a weak oscillation. Rusch and Erickson were limited to a lower energy range (200-2000 eV), where oscillation might be even less clear.

The oscillatory behavior of $\mathrm{He}^{+}$scattering by $\mathrm{La}$ is verified by subtracting the linear trend of Eq. (5) from the experimental data of Fig. 6. The results of this subtraction are shown in Fig. 7. Since qRCT is a local mechanism, the curves were plotted against $1 / v_{0}$ instead of the perpendicular velocity components. Since $v_{o}, v_{f}$ and their surface normal components are all proportional to each other, the structure of $\ln \left(\frac{s_{i}}{\frac{d \sigma_{i}}{d \Omega}}\right)$ versus $1 / v$ plots is independent on the choice of the representation of $1 / v$. The choice of $1 / v$ model only affects characteristic velocity. Representing $1 / v$ as $1 / v_{0}$ allows to compare our dependencies to literature data about qRCT, where the Godfrey-Woodruff model is often selected. What remains after the linear subtraction looks like a clear oscillation, with two minima and two maxima. It is still much less pronounced than oscillations observed for some materials [37,26], but nevertheless observable. In their original classification paper [24] Rusch and Erickson made a statement that if the range of primary ion energy would be increased, class IV (structured ion yield) may be an extension of class II (oscillatory ion yield). This agrees well with our observation.

We compared the period of oscillations in $\mathrm{He}^{+}$scattering from La to the periods of previously reported oscillations. Of course, exact values depend on the scattering geometry and also even in the inversed velocity scale oscillations do not have a constant period, increasing for larger $1 / v_{0}$. Still, average periods can be calculated for a rough comparison. We find $8 \times 10^{-7} \mathrm{~s} / \mathrm{m}$ for $\mathrm{Ga}$ and $10 \times 10^{-7} \mathrm{~s} / \mathrm{m}$ for $\mathrm{Pb}$ from Tolk [38], $9.3 \times 10^{-7} \mathrm{~s} / \mathrm{m}$ for $\mathrm{Pb}$ and

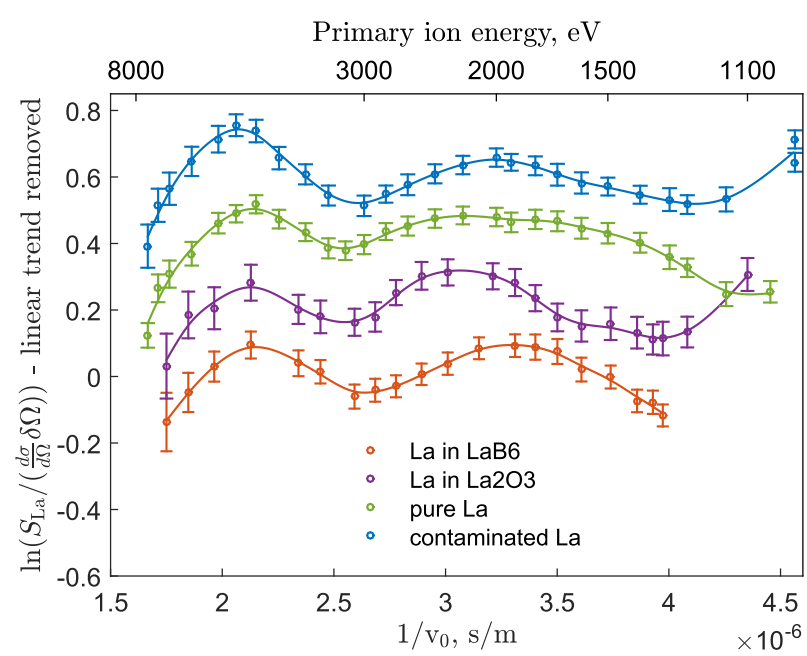

Fig. 7. Deviation from linear fit in dependence of the logarithmical normalized integrated La LEIS signal $\ln \left(\frac{S_{L a}}{\frac{d \sigma}{d \Omega} \delta \Omega}\right)$ on the inverse incident velocity of ${ }^{4} \mathrm{He}^{+}$ions $1 / v_{0}$. The curves are vertically shifted for convenience. 
$8.7 \times 10^{-7} \mathrm{~s} / \mathrm{m}$ for Ge from Erickson [39] and $6.7 \times 10^{-7} \mathrm{~s} / \mathrm{m}$ for Ge from Goebl [26]. Our data for La gives around $11 \times 10^{-7} \mathrm{~s} / \mathrm{m}$. These values are not exactly the same, but similar, which is expected from a simple quasi-molecular state model $[38,40]$.

The next step is to find out which La level actually contributes to qRCT. There are three La levels in the vicinity of the He 1s level, these are La $5 s$, La $5 p_{1 / 2}$ and $5 p_{3 / 2}$, which have binding energies of $34.3 \mathrm{eV}, 19.3 \mathrm{eV}$ and $16.8 \mathrm{eV}$, respectively. Here and later in the paper all the values for binding energies are taken from Gwyn William's table [41]. Since it is relative to the Fermi level and the work function of La is $3.5 \mathrm{eV}$ [33], the energy mismatches $\Delta E$ between these levels and He $1 \mathrm{~s}(24.6 \mathrm{eV})$ are $13.2,-1.8$ and $-4.3 \mathrm{eV} . \Delta E$ of La $5 \mathrm{~s}$ is extremely large for qRCT, but there are observed cases of very weak resonances in Sb [24], with the closest level being $4 \mathrm{~d}_{5 / 2}$, with $\Delta E$ of $11.5 \mathrm{eV}$. So there is a chance of very weak resonance with La $5 \mathrm{~s}$ too. However, most of the curves from Fig. 7 are composed of more than one oscillatory component, especially for pure La and contaminated La. To create this interference effect two resonating levels are required. If such behavior would be observed only for the contaminated La sample with multiple La valence states, but not for the pure La sample, it could be explained by two different states of 5 s with different binding energies, like La oxide and elemental La. This is, however, not the case, so this effect cannot be easily explained by interaction with $5 \mathrm{~s}$ orbitals. Another option is a double resonance with La $5 p_{1 / 2}$ and $5 p_{3 / 2}$. Energy mismatches of 1.8 and $4.3 \mathrm{eV}$ are enough for strong resonances, so in our case the reason of the weakness of the observed oscillations has to be connected with a different symmetry of the interacting $5 \mathrm{p}$ and $1 \mathrm{~s}$ orbitals. The symmetry condition for the occurence of resonances was first introduced by Rusch and Erickson [24], based on the fact that to this moment only clear oscillating ion yield curves of low energy $\mathrm{He}^{+}$scattering were observed from materials with d orbitals lying close to He 1 s.

Back in 1995 Arikawa [42] reported oscillations in $\mathrm{He}^{+}$scattering from Ta and $\mathrm{W}$, which have $4 \mathrm{f}$ levels close to He $1 \mathrm{~s}$. This finding did not become widely known in the LEIS community, maybe because the oscillatory structure was not sufficiently pronounced for the given error margin. Also the strength of the claimed oscillations was similar in both Ta and $\mathrm{W}$, although Ta has $4 \mathrm{f}_{5 / 2}$ and $4 \mathrm{f}_{7 / 2}$ within $3 \mathrm{eV}$ of He $1 \mathrm{~s}$, while for $\mathrm{W}$ the closest $4 \mathrm{f}_{7 / 2}$ orbital is as far as 11 [41]-14 eV [42] from He 1s.

The origin of the symmetry rule is a conservation of the total (molecular) symmetry during the ion-atom collision in a collinear geometry. However, to our best knowledge, a selection rule prohibiting electron transitions between $\mathrm{s}$ and $\mathrm{p}$ orbitals does not exist, and also never appears in theoretical descriptions [43]. Additionally to that, it is known in spectroscopy [44] for symmetries to be distorted in the crystal lattice, so if there is a symmetry rule it can be violated due to surface La atoms being part of a bulk sample. Therefore a bandwidth condition is possibly a more logical one than a symmetry condition. Strong $\mathrm{s} \leftrightarrow \mathrm{d}$ resonances can also be explained by a usually narrow band of the $3 \mathrm{~d}$ electrons.

The present results suggest that resonating orbitals are La $5 p$ rather than 5s. A more convincing proof would require a check on other elements which have different binding energies of these levels. We have performed a literature check for all elements which have $\mathrm{p}$ levels within $6 \mathrm{eV}$ from He 1s. Such elements were found within 4 th, 5 th and 6 th groups of the periodic table. They are shown in Table 2. The 7th group was not considered due to the practical difficulties that radioactive materials present. For most of the elements presented in Table 2 we either couldn't find any LEIS studies, or if $\mathrm{He}^{+}$scattering was done, only a single energy was used, so that no conclusions about energy dependence of $\mathrm{He}^{+}$ yield can be done. There are two exceptions: a group of lanthanides and Ba. Several lanthanides (La, Ce, Nd, Sm, Gd, Dy, Er, Yb) with "structured" ion yield were reported by Rusch and Erickson [24]. Due to the absence of actual graphs it is hard to make conclusions, but if structuring effects are similar for all of these lanthanides, it would be another indirect suggestion of the involvement of $5 p$ levels, since for the heavier lanthanides $5 \mathrm{~s}$ levels are much further from $\mathrm{He} 1 \mathrm{~s}$. The last element under consideration is Ba. Its ion yield energy dependence curves were extensively studied due to its work function related matrix effect. Unfortunately not more than 4 different energies were used by Cortenraad et al. [12] as well

Table 2

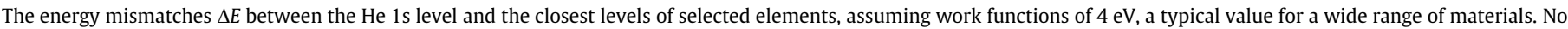
articles about LEIS were found for the elements marked by "-".

\begin{tabular}{|c|c|c|c|c|c|c|c|}
\hline \multirow{2}{*}{$\begin{array}{l}\text { Element } \\
\text { 4th period }\end{array}$} & \multicolumn{3}{|c|}{ Energy of selected levels, eV [41] } & \multicolumn{3}{|c|}{$\begin{array}{l}\text { Energy mismatch between selected levels } \\
\text { and } \mathrm{He} 1 \mathrm{~s}, \mathrm{eV}\end{array}$} & \multirow[t]{2}{*}{ LEIS studies in the literature } \\
\hline & $3 s$ & $3 \mathrm{p} 1 / 2$ & $3 \mathrm{p} 3 / 2$ & $3 s$ & $3 \mathrm{p} 1 / 2$ & $3 \mathrm{p} 3 / 2$ & \\
\hline $\mathrm{K}$ & 34.8 & 18.3 & 18.3 & 14.2 & -2.3 & -2.3 & Single $\mathrm{He}+$ energy \\
\hline $\mathrm{Ca}$ & 44.3 & 25.4 & 25.4 & 23.7 & 4.8 & 4.8 & Single $\mathrm{He}+$ energy \\
\hline 5th period & $4 \mathrm{~s}$ & $4 \mathrm{p} 1 / 2$ & $4 \mathrm{p} 3 / 2$ & $4 s$ & $4 \mathrm{p} 1 / 2$ & $4 \mathrm{p} 3 / 2$ & \\
\hline $\mathrm{Rb}$ & 30.5 & 16.3 & 15.3 & 9.9 & -4.3 & -5.3 & - \\
\hline $\mathrm{Sr}$ & 38.9 & 21.3 & 20.1 & 18.3 & 0.7 & -0.5 & - \\
\hline $\mathrm{Y}$ & 43.8 & 24.4 & 23.1 & 23.2 & 3.8 & 2.5 & Single $\mathrm{He}+$ energy \\
\hline 6th period & $5 s$ & $5 \mathrm{p} 1 / 2$ & $5 \mathrm{p} 3 / 2$ & $5 s$ & $5 \mathrm{p} 1 / 2$ & $5 \mathrm{p} 3 / 2$ & \\
\hline $\mathrm{Ba}$ & 30.3 & 17 & 14.8 & 9.7 & -3.6 & -5.8 & Few points $[34,12]$ \\
\hline La & 34.3 & 19.3 & 16.8 & 13.7 & -1.3 & -3.8 & Structured [24] \\
\hline $\mathrm{Ce}$ & 37.8 & 19.8 & 17 & 17.2 & -0.8 & -3.6 & Structured [24] \\
\hline $\operatorname{Pr}$ & 37.4 & 22.3 & 22.3 & 16.8 & 1.7 & 1.7 & - \\
\hline Nd & 37.5 & 21.1 & 21.1 & 16.9 & 0.5 & 0.5 & Structured [24] \\
\hline $\mathrm{Pm}$ & - & - & - & - & - & - & -, unstable \\
\hline $\mathrm{Sm}$ & 37.4 & 21.3 & 21.3 & 16.8 & 0.7 & 0.7 & Structured [24] \\
\hline $\mathrm{Eu}$ & 32 & 22 & 22 & 11.4 & 1.4 & 1.4 & - \\
\hline Gd & 36 & 28 & 21 & 15.4 & 7.4 & 0.4 & Structured [24] \\
\hline $\mathrm{Tb}$ & 45.6 & 28.7 & 22.6 & 25 & 8.1 & 2 & - \\
\hline Dy & 49.9 & 26.3 & 26.3 & 29.3 & 5.7 & 5.7 & Structured [24] \\
\hline Ho & 49.3 & 30.8 & 24.1 & 28.7 & 10.2 & 3.5 & - \\
\hline $\mathrm{Er}$ & 50.6 & 31.4 & 24.7 & 30 & 10.8 & 4.1 & Structured [24] \\
\hline $\operatorname{Tm}$ & 54.7 & 31.8 & 25 & 34.1 & 11.2 & 4.4 & - \\
\hline $\mathrm{Yb}$ & 52 & 30.3 & 24.1 & 31.4 & 9.7 & 3.5 & Structured [24] \\
\hline $\mathrm{Lu}$ & 57.3 & 33.6 & 26.7 & 36.7 & 13 & 6.1 & Single $\mathrm{He}+$ energy \\
\hline
\end{tabular}


as by Denier van der Gon et al. [34]. This is certainly not enough to see if weak oscillations could be present.

This table thus shows that it is unknown whether there are oscillatory ion yields for potential $\mathrm{s} \leftrightarrow \mathrm{p}$ resonating material combinations. For now there is no experimental contradiction for potential existence of $\mathrm{s} \leftrightarrow \mathrm{p}$ resonances. Dedicated measurements on more of the listed elements should be done to decide whether the $\mathrm{He} \leftrightarrow \mathrm{La}$ resonance is caused by La $5 \mathrm{p}$ or La 5 s orbitals. Also proper calculations are needed to understand why oscillations for La appear in a slightly shifted energy range compared to $\mathrm{s} \leftrightarrow \mathrm{d}$ resonances, and how strong is the dampening effect for potential $\mathrm{s} \leftrightarrow$ $p$ resonances. We leave these questions open without addressing them in this paper.

Lastly, oscillations presented in Fig. 7 differ for different compounds of La. This is another matrix effect. This time it is connected not to C-RN, but to qRCT. It is caused by a (chemical) shift in the binding energy of resonating La $5 p$ (or 5 s) levels, which is the same order of magnitude as the separation of the La $5 p$ doublet. The presence of a $5 p_{1 / 2}$ and $5 p_{3 / 2}$ doublet should only make the effect stronger because of interference of two different oscillations. Matrix effects in qRCT were previously observed for stronger oscillating elements like In [39,24] or Ga [45]. Our study, however, presents the evidence that the matrix effects in C-RN and qRCT can happen simultaneously and independently in a single sample. The described double matrix effect should not be specific only for La and was probably not discovered before only because accurate variable energy LEIS measurements in a wide energy range are seldom. Regardless of which of the La energy levels actually contribute to qRCT, there are more materials from Table 2 which will have both low work function and energy levels close to He $1 \mathrm{~s}$, therefore exhibiting similar complex behavior as La.

\section{Conclusions}

In this work we performed LEIS studies of surfaces of pure polycrystalline La films as well as its compounds, $\mathrm{LaB}_{6}$, and $\mathrm{La}_{2} \mathrm{O}_{3}$. During sputter depth profiling La thin films showed large unexpected change of $\mathrm{He}^{+}$yield in the absence of other elements in the LEIS spectra. We proved that this is a matrix effect caused by presence of subsurface $\mathrm{B}$ or $\mathrm{O}$ atoms. To study the matrix effect and enable quantification of surface composition, we measured $\mathrm{He}^{+}$yield for incident ion energies in the range from 1000 to $7500 \mathrm{eV}$.

During the investigation we found two separate matrix effects in La compounds, leading to a double matrix effect. One of the matrix effects is work function related and is associated with resonant charge transfer from the conduction band of the target to excited states of the $\mathrm{He}^{+}$ion. We also want to emphasize the fact that this matrix effect can change the La signal even if the element that is causing the matrix effect is hidden in the subsurface layer, and La is the only element visible by LEIS. This mechanism was previously used to describe other systems [12], and in this work we show its applicability to La-based surfaces as well.

Another matrix effect is related to a shift of binding energy of La bound energy levels and is associated with quasiresonant charge transfer between La and He. We suggest that quasiresonance is happening between La $5 \mathrm{p}$ and He $1 \mathrm{~s}$ orbitals, although the given data doesn't allow us to rule out the involvement of La 5s orbitals. The final answer to this question cannot be given without additional experiments on other elements. This is the first time quasiresonant charge transfer is proven to be present in La. In case of confirmation of He $1 \mathrm{~s} \leftrightarrow$ La 5 p quasiresonance it will be also be the first observation of $\mathrm{s} \leftrightarrow \mathrm{p}$ resonance in LEIS in general, deviating from the original suggestion in the literature that only $\mathrm{s} \leftrightarrow \mathrm{d}$ resonances exist. The presence of quasiresonant change transfer was detected by weak oscillations present in the energy depen- dence of the $\mathrm{He}^{+}$ion yield from La. Only the broad energy range used in this study allowed detection of the oscillations. Since most of the earlier studies of the energy dependencies were done for lower $\mathrm{He}^{+}$energies, there may be more elements with oscillatory ion yields than already reported.

Understanding the mechanisms responsible for matrix effects allowed us to quantify the amount of La on the surfaces of studied samples using the ion yield dependence on the incident ion energy and extrapolating to infinite energy. This quantification is more difficult and less precise than for the most of other elements due to two simultaneous factors contributing to the behavior of this energy dependence. Nevertheless, we showed that precise measurements over a wide energy range allow determination of the surface composition even in the presence of complex matrix effects.

\section{Acknowledgements}

We acknowledge the support of the Industrial Focus Group XUV Optics at the MESA+ Institute at the University of Twente, notably the industrial partners ASML, Carl Zeiss SMT AG, PANalytical, TNO, as well as the Province of Overijssel and NWO. We also express our gratitude to ION-TOF, especially Philipp Brüner and Dr. Thomas Grehl for cooperation and opportunity to use LEIS setup in Münster. Additionally we thank Gerard Kip for $\mathrm{LaB}_{6}$ cathode, and Jorik Houtman and Sjoerd Visser, with whom the studies of matrix effects in La began.

\section{References}

[1] V. Banine, A. Yakunin, D. Glushkov, Next generation EUV lithography: challenges and opportunities, in: 2010 International Workshop on Extreme Ultraviolet Sources, 2010, p. 14.

[2] T. Tsarfati, R. van de Kruijs, E. Zoethout, E. Louis, F. Bijkerk, Reflective multilayer optics for $6.7 \mathrm{~nm}$ wavelength radiation sources and next generation lithography, Thin Solid Films 518 (2009) 1365-1368.

[3] D.S. Kuznetsov, A.E. Yakshin, J.M. Sturm, R.W.E. van de Kruijs, E. Louis, F. Bijkerk, High-reflectance La/B-based multilayer mirror for $6 \mathrm{x}$ nm wavelength, Opt. Lett. 40 (2015) 3778.

[4] U. Bardi, Determination of the growth mechanism of overlayers on solid surfaces: a method based on combined XPS and LEIS measurements, Appl. Surf. Sci. 51 (1991) 89-93.

[5] J. Shin, D. Gay, Y.M. Sun, J.M. White, J.G. Ekerdt, Growth and characterization of ultrathin metal films for ULSI interconnects, AIP Conf. Proc. 788 (2005) 482 487.

[6] V.I.T.A. de Rooij-Lohmann, A.E. Yakshin, R.W.E. van de Kruijs, E. Zoethout, A.W. Kleyn, E.G. Keim, M. Gorgoi, F. Schäfers, H.H. Brongersma, F. Bijkerk, Enhanced diffusion upon amorphous-to-nanocrystalline phase transition in $\mathrm{Mo} / \mathrm{B} 4 \mathrm{C} / \mathrm{Si}$ layered systems, J. Appl. Phys. 108 (2010) 014314.

[7] G. Vári, L. Óvári, J. Kiss, Z. Kónya, LEIS and XPS investigation into the growth of cerium and cerium dioxide on $\mathrm{Cu}(111)$, Phys. Chem. Chem. Phys. 17 (2015) 5124-5132.

[8] Thermo Fisher Scientific, Avantage Software, 2018. <https:// xpssimplified.com/avantage_data_system.php>.

[9] J.H. Scofield, Hartree-Slater subshell photoionization cross-sections at 1254 and 1487 eV, J. Electron. Spectrosc. Relat. Phenom. 8 (1976) 129-137.

[10] S. Průsa, P. Procházka, P. Bábor, T. Šikola, R. Ter Veen, M. Fartmann, T. Grehl, P. Brüner, D. Roth, P. Bauer, H.H. Brongersma, Highly sensitive detection of surface and intercalated impurities in graphene by LEIS, Langmuir 31 (2015) 9628-9635.

[11] Y. Maeno, M. Yamamoto, S. Naito, M. Mabuchi, T. Hashino, Change in the work function of zirconium by oxidation at high temperatures and low oxygen pressures, Faraday Trans. 87 (1991) 1399.

[12] R. Cortenraad, A.W.D. van der Gon, H.H. Brongersma, S.N. Ermolov, V.G Glebovsky, Work function dependent neutralization of low-energy noble gas ions, Phys. Rev. B 65 (2002) 195414.

[13] P.J. Cumpson, M.P. Seah, Elastic scattering corrections in AES and XPS. II Estimating attenuation lengths and conditions required for their valid use in overlayer/substrate experiments, Surf. Interface Anal. 25 (1997) 430-446.

[14] H. Brongersma, M. Draxler, M. de Ridder, P. Bauer, Surface composition analysis by low-energy ion scattering, Surf. Sci. Rep. 62 (2007) 63-109.

[15] W.P.A. Jansen, A. Knoester, A.J.H. Maas, P. Schmit, A. Kytokivi, A.W.Denier v.d. Gon, H.H. Brongersma, Influence of compaction and surface roughness on lowenergy ion scattering signals, Surf. Interface Anal. 36 (2004) 1469-1478.

[16] H.R.J. ter Veen, T. Kim, I.E. Wachs, H.H. Brongersma, Applications of high sensitivity-low energy ion scattering (HS-LEIS) in heterogeneous catalysis, Catal. Today 140 (2009) 197-201. 
[17] H.D. Hagstrum, Theory of auger ejection of electrons from metals by ions, Phys. Rev. 96 (1954) 336-365.

[18] D. Godfrey, D. Woodruff, Elastic and neutralisation effects in structural studies of oxygen and carbon adsorption on $\mathrm{Ni}$ (100) surfaces studied by low energy ion scattering, Surf. Sci. 105 (1981) 438-458.

[19] R. Cortenraad, A.W. Denier van der Gon, H.H. Brongersma, S.N. Ermolov, V.G. Glebovsky, Quantification of the surface composition of low-work function surfaces using low-energy ion scattering, Surf. Interface Anal. 31 (2001) 200205.

[20] G. van Leerdam, H. Brongersma, Shielding effects in oxides studied by lowenergy ion scattering, Surf. Sci. Lett. 254 (1991) A479.

[21] A. Tolstogouzov, S. Daolio, C. Pagura, C.L. Greenwood, Dependence of scattered ion yield on the incident energy: $\mathrm{Ne}^{+}$on pure gallium and indium, Surf. Sci. 531 (2003) 95-102.

[22] S. Rund, D. Primetzhofer, S.N. Markin, D. Goebl, P. Bauer, Charge exchange of He+-ions with aluminium surfaces, Nucl. Instrum. Methods Phys. Res., Sect. B: Beam Interact. Mater. Atoms 269 (2011) 1171-1174.

[23] R. Souda, M. Aono, C. Oshima, S. Otani, Y. Ishizawa, Shadowing and focusing effects in the angular distributions of low-energy rare-gas ions scattered from solid surfaces, Surf. Sci. 179 (1987) 199-208.

[24] T.W. Rusch, R. Erickson, Energy dependence of scattered ion yields in ISS, J. Vac. Sci. Technol. 13 (1976) 374.

[25] W. Lichten, Resonant charge exchange in atomic collisions. II. Further applications and extension to the quasi-resonant case, Phys. Rev. 139 (1965) A27-A34.

[26] D. Goebl, D. Roth, D. Primetzhofer, R.C. Monreal, E. Abad, A. Putz, P. Bauer, Quasi-resonant neutralization of $\mathrm{He}+$ ions at a germanium surface, J. Phys. Condens. Matter 25 (2013) 485006.

[27] R. Nishitani, M. Aono, T. Tanaka, C. Oshima, S. Kawai, H. Iwasaki, S. Nakamura, Surface structures amd work functions of the LaB6 (100), (110) and (111) clean surfaces, Surf. Sci. 93 (1980) 535-549.

[28] M. Trenary, Surface science studies of metal hexaborides, Sci. Technol. Adv Mater. 13 (2012) 023002

[29] M. Ushio, A.A. Sadek, F. Matsuda, Comparison of temperature and work function measurements obtained with different GTA electrodes, Plasma Chem. Plasma Process. 11 (1991) 81-101.

[30] X. Xi, Z. Nie, W. Wang, J. Yang, S. Hao, Y. Guo, T. Zuo, Study on preparation and emission properties of nano-composite WLa2O3 material, Appl. Surf. Sci. 251 (2005) 134-138.
[31] M. Tanaka, S. Tashiro, H. Nishikawa, M. Ushio, Effective electrode work functions in argon gas tungsten arc during operation, Surf. Coat. Technol. 201 (2007) 5383-5386.

[32] S. Halas, T. Durakiewicz, Physical foundations of the oxide cathodes, Appl. Surf. Sci. 252 (2006) 6119-6121.

[33] J. Speight, Lange's Handbook of Chemistry, 16th ed., McGraw-Hill, New York, 2005.

[34] A.W.D. van der Gon, M.F.F.K. Jongen, H.H. Brongersma, U. van Slooten, A. Manenschijn, Thermionic cathodes studied by low-energy ion scattering spectroscopy, Appl. Surf. Sci. 111 (1997) 64-69.

[35] J.F. Ziegler, J.P. Biersack, The stopping and range of ions in matter, in: Treatise on Heavy-Ion Science, Springer US, Boston, MA, 1985, pp. 93-129.

[36] S.N. Mikhailov, R.J.M. Elfrink, J.P. Jacobs, L.C.A. van den Oetelaar, P.J. Scanlon, H. $\mathrm{H}$. Brongersma, Quantification in low-energy ion scattering: elemental sensitivity factors and charge exchange processes, Nucl. Inst. Methods Phys. Res. B 93 (1994) 149-155.

[37] D. Christensen, V. Mossotti, T. Rusch, R. Erickson, Oscillatory scattered ion yields from nonconductive materials, Nucl. Instrum. Methods 149 (1978) 587590.

[38] N.H. Tolk, J.C. Tully, J. Kraus, C.W. White, S.H. Neff, Angular dependence of oscillatory structure in low-energy ion-surface scattering, Phys. Rev. Lett. 36 (1976) 747-750.

[39] R. Erickson, D. Smith, Oscillatory cross sections in low-energy ion scattering from surfaces, Phys. Rev. Lett. 34 (1975) 297-299.

[40] R. Souda, T. Aizawa, C. Oshima, M. Aono, S. Tsuneyuki, M. Tsukada, Quasiresonant charge-exchange and reionization in low energy $\mathrm{He}^{+}$ scattering from Sn, Surf. Sci. Lett. 187 (1987) L592-L598.

[41] W.M. Haynes, CRC Handbook of Chemistry and Physics, 96th ed., 2015.

[42] T. Arikawa, O. Kishimoto, W. Aoba, Ion scattering spectroscopy experiment in collisions of $\mathrm{He}+$ ions with Ta and W targets, Jpn. J. Appl. Phys. 34 (1995) $1561-1563$.

[43] H. Shao, D.C. Langreth, P. Nordlander, Theoretical description of quasiresonant charge exchange in atom-surface collisions, Phys. Rev. B 49 (1994) 1394813965.

[44] D.A. Long, The Raman Effect: A Unified Treatment of the Theory of Raman Scattering by Molecules, vol. 8, 2002.

[45] H. Helbig, K. Orvek, Isotope effects in elastic ion-surface scattering; $\mathrm{He}+$ and $\mathrm{Ne}$ + on solid and liquid Ga, Nucl. Instrum. Methods 170 (1980) 505-508. 